doi:10.12662/2359-618xregea.v9i1.p64-73.2020

ARTIGOS

\section{ATIPICIDADES EM GRUPOS DE PESQUISA DOS INSTITUTOS FEDERAIS DO RIO DE JANEIRO - LEVANTAMENTO DOS QUANTITATIVOS EM 2018 DE ACORDO OS CRITÉRIOS DA CNPQ}

\author{
RIO DE JANEIRO FEDERAL INSTITUTES OF \\ EDUCATION, SCIENCE AND TECHNOLOGY \\ RESEARCH GROUPS - 2018 DATA SURVEY \\ ACCORDING WITH CNPQ CRITERIA
}

\section{RESUMO}

A quantidade de grupos de pesquisa dos Institutos Federais no estado do Rio de Janeiro teve um crescimento significativo nos últimos anos. No entanto, apesar dessa evolução, são necessárias avaliações de caráter qualitativo do panorama de pesquisa da Rede. Uma das formas de se avaliar isso é por meio dos critérios de atipicidade definidos pelo CNPq. Neste trabalho, foram caracterizados os grupos de pesquisa das Escolas Técnicas do RJ, Cefet-RJ, IFF e IFRJ, em relação às suas atipicidades. Os resultados, obtidos mediante de levantamento documental, análise de distribuições de frequência e estatística descritiva simples, permitiram identificar um alto grau de atipicidade dos grupos de pesquisa dessas instituições. As análises permitiram ordenar os critérios de atipicidades por maior frequência, o que destacou pontos em comum entre as organizações. Ao final, há sugestões para análise qualitativa as quais são realizadas pelos grupos de pesquisa.

Palavras-chave: Grupos de pesquisa. Atipicidades. Indicadores.

Guilherme Veloso Castello gvcastello@gmail.com Mestre em Sistemas de Gestão pela Universidade Federal Fluminense. Pesquisador do Instituto Federal Fluminense. Macaé - RJ - BR.

\footnotetext{
Allan Rodrigues Ramos allan.rr_09@hotmail.com Discente do Curso Técnico em Automação Industrial - IF Fluminense Campus Macaé. Bolsista de Iniciação Científica Júnior do CNPq. Macaé - RJ - $B R$.
}

\begin{abstract}
The number of research groups at Rio de Janeiro's Federal Institutes of Education, Science and Technology has grown significantly in the past few years. However, despite this evolution, other evaluations of the Network's research landscape are useful to provide a better understanding about this movement. One of the alternatives to evaluate this is through $\mathrm{CNPq}^{\prime}$ s atypicalities criteria. In this work, the research groups of the Rio de Janeiro's Technical Schools, Cefet-RJ, IFF and IFRJ were described in relation to their atypicalities. The results, obtained through documentary survey, frequency distribution analysis and simple descriptive statistics, allowed to
\end{abstract}


identifying a high level of atypicalities of the research groups of these institutions. The analysis also permitted to order the atypicality criteria by greater frequency, which pointed out common features between the organizations. At the end, there are suggestions for qualitative analysis which are carried out by the research groups.

Keywords: Research groups. Atypicalities. Indicators.

\section{INTRODUÇÃO}

No Brasil, o Conselho Nacional de Desenvolvimento Científico e Tecnológico (CNPq), agência do Ministério da Ciência, Tecnologia, Inovações e Comunicações (MCTIC), desempenha papel crucial na formulação e condução das políticas de ciência, tecnologia e inovação ao fomentar a pesquisa científica e tecnológica e incentivar a formação de pesquisadores brasileiros.

Uma das ações do órgão se concentra no Diretório de Grupos de Pesquisa (DGP), em que os grupos de pesquisa - conjunto de indivíduos organizados com envolvimento profissional e permanente com atividade de pesquisa cujo trabalho se organiza em torno de linhas de pesquisa (CONSELHO NACIONAL DE DESENVOLVIMENTO CIENTÍFICO E TECNOLÓGICO, 2018a) - são cadastrados e têm suas informações registradas sobre sua estrutura. Com essas informações, que estão acessíveis no site do Diretório de Grupos de Pesquisa, é possível caracterizar, monitorar e avaliar a produção científica desses grupos. Por meio desses dados, o trabalho de Miano et al. (2017), caracterizou os grupos de pesquisa dos Institutos Federais do Estado do Rio de Janeiro, Cefet- RJ, IFF e IFRJ, entre 2008 e 2016 sob os diversos aspectos como o número de pesquisadores, doutores, grupos de pesquisa e a evolução quantitativa da produção bibliográfica. O trabalho também mostrou que, no período, os grupos de pesquisa aumentaram $223,81 \%$, o número de doutores $375,46 \%$ e o número de pesquisadores $435,14 \%$. Outros trabalhos sobre a rede federal de educação técnica e tecnológica, assim como outras Instituições de Ensino Superior (IES) federais, também já abordaram temas, como produção bibliográfica (PERUCCHI; GARCIA, 2012; MIANO et al., 2017), caracterização de grupos de pesquisa (MOTA; CARDOSO; SANTOS, 2010), redes de colaboração entre pesquisadores em áreas de pesquisa específicas e suas produções (SANTANA et al., 2014), alinhamento de políticas institucionais relacionadas à atividade de pesquisa (MATOS, 2014), entre outros.

Complementando a avaliação quantitativa dos grupos mencionados, cabe avançar na análise dos aspectos qualitativos dos Institutos Federais do Rio de Janeiro. Neste sentido, o presente trabalho caracteriza os grupos de pesquisa dos Institutos Federais do Estado do Rio de Janeiro, a partir dos critérios de atipicidade definidos pelo CNPq. Esses critérios se definem em grande parte, mas não somente, pelo afastamento dos grupos de pesquisa do perfil estatístico médio e, a partir desses critérios, que incluem grupos formados por apenas um pesquisador, grupos sem estudantes, grupos com muitos pesquisadores, entre outros, os grupos de pesquisa são classificados ou não como atípicos, permitindo uma avaliação de características desses grupos e suas contribuições.

Os resultados obtidos mostram que há um alto grau de atipicidade nos grupos dos Institutos Federais do Rio de Janeiro, e algumas ocorrem com maior frequência, como a pouca inclusão de técnicos nos grupos de pesquisa, enquanto ocorre grande participação de discentes. Por fim, o trabalho contribui para a avaliação e a caracterização dos grupos de pesquisa sob os critérios de atipicidades adotados pelo $\mathrm{CNPq}$ e propõe outras formas de avaliação qualitativas a serem abordadas nesse e em outros contextos.

\section{REVISÃO DE LITERATURA}

Antes de apresentarmos os dados relativos ao levantamento de atipicidades dos grupos de pesquisa das organizações escolhidas, devemos introduzir o histórico de desenvolvimento da Rede Federal de Educação Profissional e Tecnológica, no país e no estado do RJ, descrevendo as mudan- 
ças de princípios de atuação - com a incorporação da indissociabilidade entre ensino, pesquisa e extensão, a expansão de sua estrutura e outros fatores relevantes para a compreensão do tema. Em seguida, serão apresentados os conceitos definidos pelo $\mathrm{CNPq}$ para os grupos de pesquisa, alguns dados do DGP e informações úteis para posterior análise das atipicidades encontradas.

\subsection{REDE FEDERAL DE EDUCAÇÃO PROFISSIONAL, CIENTÍFICA E TECNOLÓGICANO ESTADO DO RJ}

A Educação Profissional e Tecnológica orienta processos de formação com base nas premissas da integração e da articulação entre ciência, tecnologia, cultura e seus conhecimentos específicos no desenvolvimento da investigação científica, autonomia e dos saberes da laboralidade, que se traduzem nas ações de ensino, pesquisa e extensão, contribuindo para o desenvolvimento socioeconômico com destaque para os focos locais e regionais (BRASIL, 2010; INSTITUTO FEDERAL DE EDUCAÇÃO, CIÊNCIA E TECNOLOGIA FLUMINENSE, 2017; INSTITUTO FEDERAL DE EDUCAÇÃO, CIÊNCIA E TECNOLOGIA DO RIO DE JANEIRO, 2017; CEFET-RJ, 2017). A Rede Federal de Educação Profissional, Científica e Tecnológica, criada em 2008, com a publicação da Lei $\mathrm{n}^{\circ} 11.892$, possui grande destaque nacional em vários aspectos. Sua capilaridade e princípio institucional de interiorização do conhecimento auxiliam na promoção do desenvolvimento socioeconômico de diversas regiões, incluindo determinadas localidades onde a oferta de formação técnica e superior era pouca ou inexistente (MOTA; CARDOSO; SANTOS, 2010). No Estado do
Rio de Janeiro, essa Rede é composta por quatro instituições: Instituto Federal de Educação, Ciência e Tecnologia Fluminense (IFF), Centro Federal de Educação Tecnológica Celso Suckow da Fonseca (CEFET/RJ), Instituto Federal de Educação, Ciência e Tecnologia do Rio de Janeiro (IFRJ) e Colégio Pedro II.

Neste trabalho, para os fins desta pesquisa, um recorte foi feito nesta Rede. O Colégio Pedro II, apesar de fazer parte da Rede Federal de Educação Profissional, Científica e Tecnológica e de desenvolver, dentro de duas dependências, grupos de pesquisa, acaba por se distanciar do perfil de grupos de pesquisa das demais instituições, por se concentrar no ensino fundamental e médio. Nas demais instituições, grande parte dos grupos de pesquisa estão concentrados na área de Engenharia, por exemplo, o que não acontece no Pedro II. Dessa forma, optou-se por não incluir essa instituição no levantamento, apesar de sua reconhecida referência no ensino fundamental e médio e por fazer parte da Rede.

Estabelecido esse recorte, essas instituições atuam em três eixos principais inter-relacionados: ensino, pesquisa e extensão, com o princípio de verticalização do ensino. Com isso, os Institutos Federais apresentam não apenas ensino de nível técnico, mas também graduações, pós-graduações lato e stricto sensu. A grande expansão da Rede nos seus primeiros dez anos, norteada por esses princípios de atuação, estimulou o crescimento relativo ao número de docentes doutores em relação ao total desses profissionais nas Instituições de Ensino Superior (IES) do estado do Rio de Janeiro. De 2008 a 2015, o percentual de professores doutores nos três Institutos Federais cresceu de uma participação de $1,66 \%$ para quase $4 \%$ em relação ao total de docentes doutores em IES do Estado do RJ.

Tabela 1 - Número de IES e de Docentes Doutores no Estado do Rio de Janeiro

\begin{tabular}{cccccccc}
\hline \multicolumn{3}{c}{ Instituições de Ensino Superior } & \multicolumn{4}{c}{ Docentes e Doutores } \\
\hline Ano & Total & IF e Cefet & Percentual & Ano & Total & IF e Cefet & Percentual \\
\hline 2008 & 136 & 3 & $2,21 \%$ & 2008 & 9.832 & 163 & $1,66 \%$ \\
2010 & 139 & 3 & $2,16 \%$ & 2010 & 12.248 & 253 & $2,07 \%$ \\
2014 & 137 & 3 & $2,19 \%$ & 2014 & 14.716 & 511 & $3,47 \%$ \\
2015 & 137 & 3 & $2,19 \%$ & 2015 & 15.543 & 612 & $3,94 \%$ \\
\hline
\end{tabular}

Fonte: elaboração dos autores com base em Instituto Nacional de Estudos e Pesquisas Educacionais Anísio Teixeira - INEP (2008, 2010, 2014, 2015). 
Os números da tabela 2 fornecem parâmetros para o dimensionamento da Rede Federal de Educação Profissional, Científica e Tecnológica no Estado do RJ em 2016:
Estes censos são realizados por meio do Diretório dos Grupos de Pesquisa (DGP), um banco de dados dos grupos de pesquisa em atividade no País (CONSELHO NACIONAL DE

Tabela 2 - Dados sobre os Institutos Federais no RJ, em 2016

\begin{tabular}{cccc}
\hline Quantidade & IFF & IFRJ & Cefet - RJ \\
\hline Técnicos-Administrativos & 738 & 852 & 628 \\
Docentes & 975 & 987 & 890 \\
Docentes com Doutorado & 204 & 379 & 311 \\
Estudantes Matriculados & 22.405 & 16.509 & 14.368 \\
Campi & 14 & 16 & 8 \\
\hline
\end{tabular}

Fonte: elaboração dos aurores com base em Relatórios de Gestão do IFF, IFRJ e Cefet-RJ (2017).

\subsection{GRUPOS DE PESQUISA}

De acordo com o Conselho Nacional de Desenvolvimento Científico e Tecnológico (2018a), um grupo de pesquisa é definido como um conjunto de indivíduos organizados hierarquicamente em torno de uma ou, eventualmente, duas lideranças, em que o critério para a liderança do grupo é a experiência e o destaque no terreno científico e tecnológico. Espera-se que esse líder seja habituado às atividades de pesquisas, produção bibliográfica e tecnológica. Sobre o grupo, este se organiza em torno de linhas comuns de pesquisa, com elevado grau de especificidade.

O CNPq realiza censos bianuais para avaliação da evolução das atividades de pesquisa. Em 2010, havia, no Brasil, 27.523 grupos de pesquisa; já o último censo, realizado em 2016, revelou a existência de 37.640 grupos no país (CONSELHO NACIONAL DE DESENVOLVIMENTO CIENTÍFICO E TECNOLÓGICO, 2018b), sendo estes bem distribuídos nas regiões geográficas do Brasil, de acordo com a tabela 3.
DESENVOLVIMENTO CIENTÍFICO ETECNOLÓGICO, 2018b), criado para armazenar e disponibilizar informações sobre os grupos de pesquisa, tais como: linhas de pesquisa, membros e suas titulações, entre outros. Os grupos de pesquisa, apesar de estarem presentes em diversas instituições, estão mais presentes em IES, que representam grande parte dos grupos cadastrados no Brasil (CONSELHO NACIONAL DE DESENVOLVIMENTO CIENTÍFICO E TECNOLÓGICO, 2018a). No estado do Rio de Janeiro, os institutos da Rede Federal vêm crescendo em participação relativa, tanto em número de pesquisadores, como no número de grupos de pesquisa (MIANO et al., 2017).

Tabela 3 - Distribuição dos Grupos de Pesquisa Segundo a Região Geográfica - 2018

\begin{tabular}{cccc}
\hline Região & Grupos & $\%$ & $\%$ Acumulado \\
\hline Sudeste & 16.009 & 42,5 & 42,5 \\
Sul & 8.637 & 23 & 65,5 \\
Nordeste & 7.713 & 20,5 & 86 \\
Centro-Oeste & 2.899 & 7,7 & 93,7 \\
Norte & 2.382 & 6,3 & 100 \\
\hline Total & 37.640 & 100 & - \\
\hline
\end{tabular}

Fonte: DGP, 2018. Adaptado de CNPq (2018a). 
Tabela 4 - Grupos de Pesquisa e Pesquisadores no Estado do RJ

\begin{tabular}{ccccccc}
\hline \multicolumn{3}{c}{ Grupos de Pesquisa } & \multicolumn{3}{c}{ Pesquisadores } \\
\hline Ano & Total RJ & IF e Cefet & Percentual & Total RJ & IF e Cefet & Percentual \\
\hline 2008 & 2.779 & 63 & $2,27 \%$ & 13.418 & 276 & $2,06 \%$ \\
2010 & 3.313 & 81 & $2,44 \%$ & 16.478 & 452 & $2,74 \%$ \\
2014 & 4.147 & 126 & $3,04 \%$ & 22.802 & 927 & $4,07 \%$ \\
2016 & 4.360 & 141 & $3,23 \%$ & 25.271 & 1.201 & $4,75 \%$ \\
\hline
\end{tabular}

Fonte: (MIANO et al., 2017, p. 62).

Cabe ressaltar que esse crescimento é superior com delimitação no estado em questão. Caso sejam comparadas taxas de outros estados, o crescimento relativo dos IFs do estado do Rio de Janeiro é menor, tanto com relação às IES (PERUCCHI; GARCIA, 2012) como a Rede Federal de Educação Profissional e Tecnológica como um todo (MOTA; CARDOSO; SANTOS, 2010). No entanto, mais uma ressalva é necessária, visto que há grande impacto dos números iniciais absolutos. Não somente os institutos federais do RJ, como as IES da região Sudeste possuem grande peso nacional, com orçamentos maiores em relação a muitos estados. Assim sendo, mesmo com maiores números absolutos tanto de frequência, como de taxa, os desempenhos relativos podem ser menores (MIANO et al., 2017).

Para uma melhor compreensão do cenário, além da análise quantitativa dos números absolutos e relativos, fazem-se necessárias outras análises. Como foi exposto, o objetivo do presente trabalho é averiguar as atipicidades totais e por critério dos institutos federais abordados, IFF, Cefet- RJ e IFRJ.

\section{METODOLOGIA}

Esta pesquisa se caracteriza como descritiva, sendo um levantamento documental com base nos dados disponíveis no DGP do CNPq. Para atingir os objetivos da pesquisa, em primeiro momento, era necessário o levantamento de tipos e quantidade de atipicidades dos grupos de pesquisa das três instituições: Instituto Federal Fluminense, Centro Federal de Educação Tecnológica Grupos de Pesquisa Celso
Suckow da Fonseca e Instituto Federal do Rio de Janeiro. Não se fez distinção entre grandes áreas do conhecimento, ou seja, as classificações encontradas dizem respeito à totalidade de grupos. Os dados, no geral, serão analisados com base em estatísticas descritivas simples, tais como média, moda e distribuição relativa.

Segundo o próprio CNPq, a definição de grupo atípico é:

É considerado grupo atípico aquele cujo perfil apresenta afastamento estatístico relevante em relação ao perfil médio dos grupos, observado no Diretório. As atipicidades devem ensejar uma melhor análise dos dados do grupo por parte do Dirigente de Pesquisa no momento da certificação. [...] Tendo o Dirigente esclarecido com o líder os motivos da atipicidade e certificado o grupo, este pode continuar atípico sem que isso lhe traga qualquer prejuízo (CONSELHO NACIONAL DE DESENVOLVIMENTO CIENTÍFICO E TECNOLÓGICO, 2018a, online).

Esta classificação justifica a escolha dos critérios de atipicidade, no entanto, não os define. Para esta classificação, o Conselho Nacional de Desenvolvimento Científico e Tecnológico (2018a) faz a conferência das seguintes características:

a) grupos unitários (formados por apenas um pesquisador);

b) grupos sem estudantes;

c) grupos sem técnicos;

d) grupos com mais de dez pesquisadores;

e) grupos com mais de dez linhas de 
pesquisa;

f) grupos cujo o líder não é doutor;

g) grupos sem doutores no conjunto de pesquisadores;

h) pesquisadores que participam de quatro ou mais grupos e;

i) estudantes que participam de dois ou mais grupos.

As fontes de dados utilizadas foram os relatórios do DGP "Casos Atípicos em Grupos Certificados" disponíveis aos diretores sistêmicos de pesquisa de cada um dos institutos, no próprio site do DGP. Cabe ressaltar que muitos campi desses Institutos possuem Diretores e/ou Coordenadores de Pesquisa. No entanto, esses relatórios são apenas disponíveis aos Pró-reitores de Pesquisa ou os diretores sistêmicos a eles diretamente vinculados. Os dados utilizados para a análise; portanto, são provenientes de relatórios oficiais gerados pelo sistema do CNPq, que compila os dados do currículo Lattes e do DGP.

Nessa tela são disponíveis quatro tipos de consultas: Grupos com mais de 10 pesquisadores ou mais de 10 linhas; grupos unitários, sem estudantes ou sem técnicos; grupos cujo líder não é doutor ou grupos sem doutor, pesquisadores que participam de quatro ou mais grupos ou estudantes em dois ou mais grupos.

No entanto, os relatórios mostram o número absoluto de atipicidades por critério de cada instituição. Assim sendo, esses conjuntos podem possuir interseções, o que dificulta a quantificação do número total de grupos atípicos. "Para essa medição, é necessário acessar a tela "Grupos de Pesquisa da sua Instituição", na qual consta a listagem de todos os grupos institucionalizados - aqueles com algum tipo de atipicidade são marcados com o ícone “!”.

Deve ser destacado que esses indicadores possuem pouca relação com a produção dos grupos. Por exemplo, um grupo típico pode apresentar baixíssima cooperação em artigos entre seus membros enquanto que um atípico apresente uma rede interna muito forte. Em outras palavras, o primeiro pode configurar-se como típico apesar de, efetivamente, pouco refletir em termos práticos em um efetivo trabalho em grupo. Algumas considerações acerca da análise desse tipo de dados podem ser encontradas em (SANTANA et al., 2014).

Por fim, com os dados sobre atipicidades em mão, procederam-se às análises dos dados obtidos, de maneira isolada, cruzando-os com dados relativos à produção bibliográfica e à distribuição entre as grandes áreas do conhecimento.

\section{RESULTADOS}

A tabela 5 abaixo lista a frequência relativa de atipicidades por critério, para cada instituição, assim como consolida o percentual total de grupos atípicos por organização.

Tabela 5 - Frequências Relativas de Atipicidades

\begin{tabular}{cccc}
\hline Critérios & IFF & IFRJ & Cefet - RJ \\
\hline Grupos unitários & $8,82 \%$ & $6,82 \%$ & $17,5 \%$ \\
Sem estudante & $5,88 \%$ & $5,68 \%$ & $5,00 \%$ \\
Sem técnico & $76,47 \%$ & $62,50 \%$ & $70,00 \%$ \\
Pesquisadores em mais de 4 & - & - & - \\
Estudantes em mais de 2 & - & - & - \\
Mais de 10 pesquisadores & $5,88 \%$ & $0,00 \%$ & $2,50 \%$ \\
Mais de 10 linhas & $85,29 \%$ & $0,00 \%$ & $75,00 \%$ \\
Grupos sem doutor & $0,00 \%$ & $0,00 \%$ & $0,00 \%$ \\
Líder sem doutorado & $0,00 \%$ & $0,00 \%$ & $0,00 \%$ \\
\hline Número Total de Grupos Atípicos & $88,24 \%$ & $82,95 \%$ & $85,00 \%$ \\
\hline
\end{tabular}

Fonte: Relatórios de Atipicidades no DGP. Elaboração dos autores. 
No primeiro critério, grupos unitários, a contagem realizada pelo CNPq contabiliza apenas os pesquisadores cadastrados, ou seja, os estudantes vinculados ao grupo não são computados. Quase a totalidade dos grupos unitários é composta pelo pesquisador e pelos estudantes que orienta (CONSELHO NACIONAL DE DESENVOLVIMENTO CIENTÍFICO E TECNOLÓGICO, 2018a), o que pode vir a demonstrar um interesse individual de pesquisa pouco articulado com os interesses de seus pares, pelo menos, ao ponto de se estabelecer uma relação formal de articulação. Nesse ponto, o Cefet-RJ apresenta uma frequência relativa duas vezes superior ao do IFF e do IFRJ. São $17,5 \%$ de casos atípicos, contra $8,8 \%$ e $6,8 \%$ respectivamente.

Uma das finalidades precípuas da atuação dos Institutos Federais, a partir da promulgação e por definição da Lei 11.892 de 2008, é a indissociabilidade entre ensino, pesquisa e extensão. Quanto à atipicidade relacionada à inserção de estudantes nos grupos de pesquisa em mais de dois grupos, todos os institutos apresentaram frequências baixas e bem próximas, entre 5 e $6 \%$. Esse é um bom indicador, que retrata a inclusão dos discentes nos projetos, o que contribui para sua formação acadêmica ou mesmo profissional.

Em outras palavras, pode indicar uma associação entre o ensino e a pesquisa. Cabe ressaltar que esse é um dos critérios com menor frequência observada. Em contraste com relação à ausência de técnicos nos grupos de pesquisa, os três institutos apresentam altos índices, o IFF de $76,47 \%$, o IFRJ de $62,50 \%$ e o Cefet-RJ 70,00\%. Isso mostra que grande parte do conhecimento que, potencialmente, possuem os técnicos sobre os processos e relações formais e informais em suas respectivas organizações não está presente nos grupos de pesquisa, ou seja, há um grande potencial de contribuição para a pesquisa sendo pouco explorado pelos grupos. Talvez também possa ser sugerido um esforço institucional nesse sentido. Os critérios "pesquisadores em mais de quatro grupos" e "estudantes em mais de dois grupos de pesquisa”, infelizmente, apresen- taram inconsistências nos seus valores, sendo assim, não puderam ser avaliados.

A discussão sobre a influência da quantidade de pessoas alocadas em um grupo de pesquisa sobre sua produtividade é bem diversa (CUMMINGS et al., 2013; COOK; GRANGE; EYERE-WALKER, 2015). O padrão definido pelo CNPq como "típico" indica equipes com menos de dez integrantes. Em todos os institutos, esse índice é baixo (IFF aproximadamente $6 \%$ e Cefet-RJ 2,5\%), com destaque ao IFRJ com frequência zero.

Na pesquisa de Cook, Grange e Eyere-Walker (2015), ao analisar grupos de pesquisa da área de ciências biológicas no Reino Unido, verificou-se uma relação positiva entre a quantidade de pessoas nos grupos de pesquisa e a produção de artigos. No entanto, essa relação se mostrou bem fraca e, em termos práticos, o aumento na quantidade de pessoas em grupos de pesquisa reflete em um aumento ínfimo na produção. De certo modo, o trabalho reflete a falta de consenso sobre a relação das variáveis, ao citar outras fontes que concluem correlações tanto positivas e negativas.

No entanto, para uma melhor avaliação da influência do tamanho do grupo em sua produtividade e relevância, esse aspecto deve ser avaliado conjuntamente com a heterogeneidade de seus componentes - tanto quanto na área de atuação como na instituição de vinculação. Ao avaliar os impactos quantitativos nos indicadores de produção pelas variáveis tamanho e heterogeneidade do grupo, Cummings et al. (2013) concluem que a produtividade dos grupos cresce com o maior número de participantes, mas que isso depende do seu grau de heterogeneidade. Grupos grandes e heterogêneos possuem crescimento marginal reduzido, implicando uma produtividade média decrescente, enquanto pequenos grupos diversificados são, comparativamente, mais produtivos.

Algumas justificativas são a crescente dificuldade em manter a coesão grupal, visto que mais participantes e maior heterogeneidade levam à redução da identificação do grupo como um todo, gerando maiores custos para 
a manutenção da motivação e o alinhamento de esforços. A diversidade de perspectivas e competências estimula a inovação, mas o do aumento número de integrantes, a partir de um determinado ponto, passa a implicar esses custos de gerenciamento que sobressaem sobre os de produção (CUMMINGS et al., 2013).

De acordo com o CNPq, algumas das características básicas dos grupos de pesquisa são alinhamento em torno de linhas comuns de pesquisa, com compartilhamento de instalações e equipamentos, em que a base de organização da hierarquia é experiência, destaque e liderança no terreno científico ou tecnológico. Um grupo com mais de dez linhas temáticas de pesquisa, provavelmente, apresenta uma dispersão que dificulta o destaque e a experiência em todas as linhas, assim como o uso comum de equipamentos e instalações.

Em um grupo não atípico em número de integrantes, mas atípico no número de linha de pesquisa, ocorre a situação na qual há mais de uma linha de pesquisa por pesquisador. Nos dados encontrados, esse critério apresenta grandes disparidades: enquanto o Cefet-RJ e o IFF apresentam elevados índices, superiores a três quartos do total, o IFRJ não apresenta nenhuma atipicidade. Apesar de esse critério demonstrar um possível indício de heterogeneidade, essa relação não é causal, visto que pode haver várias linhas de pesquisa em uma área de atuação (CUMMINGS et al., 2013).

Por último, quanto aos critérios "grupos com líder sem doutorado" e "grupos sem doutores no conjunto de pesquisadores", os três institutos não apresentam nenhuma atipicidade. Esse dado, analisado em conjunto com o crescimento absoluto do número de doutores de aproximadamente $375 \%$ nessas instituições e o aumento relativo no estado do RJ de 1,66 para 3,94\%, demonstra o engajamento desses doutores nos grupos de pesquisa.

\section{CONCLUSÕES}

O eixo Pesquisa, como princípio de atuação da Rede Federal de Educação Profissional,
Científica e Tecnológica, vem, paulatinamente, ganhando significância nos institutos que a compõe. No entanto, ainda há carência de estudos que avaliem a trajetória quantitativa e qualitativa dessa atividade.

Como citado, o número de pesquisadores, os grupos de pesquisa e a produção bibliográfica apresentaram significativos crescimentos, o que leva a avaliar qualitativamente os grupos de pesquisa e suas produções. Neste trabalho, buscou-se apresentar um perfil dos grupos de pesquisa, com relação aos critérios de atipicidade definidos pelo CNPq. Tendo em vista apenas o conjunto das três organizações abordadas, o IFRJ apresentou os melhores resultados em termos de atipicidade de seus grupos. O instituto teve as menores frequências relativas em todos os critérios, exceto em "grupos com mais de dez pesquisadores". Essa posição ganha relevância ao se considerar que $57 \%$ do total de grupos da Rede no Estado do RJ são do IFRJ e que as três instituições também contam com o menor valor relativo de atipicidades.

Um ponto evidente na análise foi a alta frequência relativa de atipicidades dos grupos, em um ou mais critérios, que é de $88 \%$ no IFF, $85 \%$ no Cefet-RJ e $73 \%$ no IFRJ. No entanto, como na pesquisa bibliográfica realizada, não foram encontrados dados de outros institutos da Rede Federal de Educação Profissional, Científica e Tecnológica, nem de IES públicas ou privadas, não é possível verificar quão significativo são esses graus de desvio dessas quantidades em relação às médias institucionais - ou mesmo se há um desvio relevante. Mas pode-se afirmar que é uma grande quantidade de desvios tendo em vista os padrões do CNPq.

Para uma compreensão mais completa dos grupos de pesquisa desses institutos, uma série de indicadores complementares são, potencialmente, produtivos. A produção bibliográfica deles, como citado anteriormente, apresentou um expressivo crescimento quantitativo. No entanto, seria útil verificar o grau de impacto desses trabalhos - uma medida qualitativa - com dados, tais como, levantamento do número de citações na base do Institute for 
Scientific Information (ISI) - Science Citation Index; número de citações no métricas no Google Acadêmico (GoogleScholar); índice h (hindex) no Scopus; entre outros.

Ademais, também são necessárias pesquisas que busquem explicar as razões dessas diferenças. Por exemplo, estudos sobre redes de colaboração apontam que a produção dos grupos se caracteriza, majoritariamente, por estudos individuais, o que, por sua vez, indica que esses possam estar mais direcionados para a formação de novos pesquisadores do que para uma efetiva colaboração interna (SANTANA et al., 2014). No presente trabalho, não foi realizada nenhuma estimativa do grau de colaboração interna ou externa dos grupos.

\section{REFERÊNCIAS}

BRASIL. Ministério da Educação. Instituto Federal de Educação, Ciência e Tecnologia - um novo modelo em educação profissional e tecnológica: concepção e diretrizes. Brasília, 2010.

CENTRO FEDERAL DE EDUCAÇÃO TECNOLÓGICA CELSO SUCKOW DA FONSECA (CEFET-RJ). Prestação de Contas Ordinárias Anual - Relatório de Gestão do Exercício de 2016. Rio de Janeiro, 2017.

CONSELHO NACIONAL DE DESENVOLVIMENTO CIENTÍFICO E TECNOLÓGICO (CNPq). Diretório de Grupos de Pesquisa. Disponível em: http://lattes.cnpq.br/web/dgp/ glossario;jsessionid $=$ XXifbN6oz4+E-8360ZjkOCoC.undefined?p_p_id=54_INSTANCE_ QoMcDQ9EVoSc\&p_p_lifecycle $=0 \& p \_p \_s t a-$ te $=$ normal\&p_p_mode $=$ view\&p_p_col_id $=-$ column-3\&p_p_col_count $=1 \&$ \&4_INSTANCE QoMcDQ9EVoSc struts action $=\% 2$ Fwiki_display\%2Fview\&_54_INSTANCE_QoMcDQ9EVoSc nodeName=Main\& 54 INSTANCE_QoMcDQ9EVoSc_title=Grupo+de+pesquisa. Acesso em: 1 mar 2018a.

CONSELHO NACIONAL DE DESENVOLVIMENTO CIENTÍFICO E TECNOLÓGICO
(CNPq). O CNPq. Disponível em: http://lattes.cnpq.br/web/dgp/home . Acesso em: 2 abr. 2018b.

COOK, I.; GRANGE, S.; EYERE-WALKER, A. Research groups: How big should they be? 2015. Disponível em: https://peerj.com/articles/989/. DOI 10.7717/peerj.989 2015.

CUMMINGS, J. N. et al. Group Heterogeneity Increases the Risks of Large Group Size: a longitudinal study of productivity in research groups. Psychological Science, v. 24, n. 6, p. 880-890, 2013.

INSTITUTO FEDERAL DE EDUCAÇÃO, CIÊNCIA E TECNOLOGIA DO RIO DE JANEIRO (IFRJ). Relatório de Gestão - Exercício 2016. Rio de Janeiro, 2017.

INSTITUTO FEDERAL DE EDUCAÇÃO, CIÊNCIA E TECNOLOGIA FLUMINENSE (IFF). Prestação de Contas Ordinária Anual - Relatório de Gestão do Exercício de 2016. Campos dos Goytacazes, 2017.

INSTITUTO NACIONAL DE PESQUISAS EDUCACIONAIS ANÍSIO TEIXEIRA. Ministério da Educação (INEP/MEC). Sinopse Estatística da Educação Superior 2008. Brasília, 2008.

INSTITUTO NACIONAL DE PESQUISAS EDUCACIONAIS ANÍSIO TEIXEIRA. Ministério da Educação (INEP/MEC). Sinopse Estatística da Educação Superior 2010. Brasília, 2010.

INSTITUTO NACIONAL DE PESQUISAS EDUCACIONAIS ANÍSIO TEIXEIRA. Ministério da Educação (INEP/MEC). Sinopse Estatística da Educação Superior 2014. Brasília, 2014.

INSTITUTO NACIONAL DE PESQUISAS EDUCACIONAIS ANÍSIO TEIXEIRA. Ministério da Educação (INEP/MEC). Sinopse 
Estatística da Educação Superior 2015. Brasília, 2015.

MATOS, L. B. O Crescimento do Instituto Federal de Brasília com Interesse no Progresso da Pesquisa Tecnológica Institucional. 2014. 105 f. Dissertação (Mestrado em Educação) - Universidade de Brasília, Brasília, 2014.

MIANO, V. Y. et al. Grupos de Pesquisa dos Institutos Federais no RJ: crescimento do eixo Pesquisa e suas áreas. Revista de Ciência e Inovação, v. 2, p. 57-67, 2017.

MOTA, L. M.; CARDOSO, E. A.; SANTOS, L. S. Uma Imagem atual da atividade de pesquisa na Rede Federal de Educação Profissional Científica e Tecnológica. In: CONGRESSO DE PESQUISA E INOVAÇÃO DA REDE NORTE E NORDESTE DE EDUCAÇÃO TECNOLÓGICA, 5., Maceió. Anais [...]. Maceió, 2010.

PERUCCHI, V.; GARCIA, J. C. R. Indicadores de produção dos grupos de pesquisa do Instituto Federal de Educação, Ciência e Tecnologia da Paraíba. Revista Brasileira de Biblioteconomia e Documentação, v. 8, p. 51- 64, 2012.

SANTANA, G. A. et al. Indicadores dos grupos de pesquisa da área de gestão da informação na região nordeste: um enfoque para a colaboração em artigos de periódicos. Em Questão, v. 20, p. 229-252, 2014. 